\title{
Quercetin Protects Primary Human Osteoblasts Exposed to Cigarette Smoke through Activation of the Antioxidative Enzymes HO-1 and SOD-1
}

Karl F. Braun, ${ }^{1}$ Sabrina Ehnert, ${ }^{2}$ Thomas Freude, ${ }^{2}$ José T. Egaña, ${ }^{3,4}$ Thilo L. Schenck, ${ }^{3}$ Arne Buchholz, ${ }^{1}$ Andreas Schmitt, ${ }^{1}$ Sebastian Siebenlist, ${ }^{1}$ Lilianna Schyschka, ${ }^{1}$

Markus Neumaier, ${ }^{1}$ Ulrich Stöckle, ${ }^{2}$ and Andreas K. Nussler ${ }^{1,2}$

${ }^{1}$ Department of Traumatology, MRI, Techincal University of Munich, 80333 Munich, Germany

${ }^{2}$ Department of Traumatology, University of Tübingen, Schnarrenbergstrße 95, 72076 Tübingen, Germany

${ }^{3}$ Department of Plastic Surgery and Hand Surgery, Techincal University of Munich, 80333 Munich, Germany

${ }^{4}$ FONDAP Center for Genome Regulation, Faculty of sciences, University of Chile, Santiago, Chile

Received 29 August 2011; Accepted 12 October 2011

Academic Editors: N. Chattopadhyay and D. Nieman

Smokers frequently suffer from impaired fracture healing often due to poor bone quality and stability. Cigarette smoking harms bone cells and their homeostasis by increased formation of reactive oxygen species (ROS). The aim of this study was to investigate whether Quercetin, a naturally occurring antioxidant, can protect osteoblasts from the toxic effects of smoking. Human osteoblasts exposed to cigarette smoke medium (CSM) rapidly produced ROS and their viability decreased concentration- and time-dependently. Co-, pre- and postincubation with Quercetin dose-dependently improved their viability. Quercetin increased the expression of the anti-oxidative enzymes heme-oxygenase- (HO-) 1 and superoxide-dismutase- (SOD-) 1. Inhibiting HO-1 activity abolished the protective effect of Quercetin. Our results demonstrate that CSM damages human osteoblasts by accumulation of ROS. Quercetin can diminish this damage by scavenging the radicals and by upregulating the expression of HO-1 and SOD-1. Thus, a dietary supplementation with Quercetin could improve bone matter, stability and even fracture healing in smokers.

KEYWORDS: Primary human osteoblasts, cigarette smoke medium, oxidative stress, Quercetin, heme-oxygenase-1. 


\section{INTRODUCTION}

Bone, as one of the most metabolically active tissues of the body, undergoes a constant and complex process of remodeling throughout life. This remodeling cycle is composed of four sequential phases. Activation precedes resorption followed by reversal and formation. Osteoblasts in particular play a key role in this cycle. Derived from osteoprogenitor cells they rise from self-renewing, pluripotent stem cells. Their main function is to produce new bone matrix and, as osteocytes, to support the bone structure itself. Impairment of osteoblasts thus can lead to several dysfunctions. Among them is delayed fracture healing, osteoporosis or an increased rate of pseudarthrosis.

Tobacco smoking is a major health risk. Cigarette smoke consists of more than 6000 molecular species, of which over 150 are known toxic compounds contributing to the pathogenesis of a variety of diseases, for example, cancer and cardiovascular and pulmonary diseases [1]. As those diseases account for up to $20 \%$ of precocious deaths in the USA, they are focus of many research groups. Upon that, in the past years several studies have described the negative effects of cigarette smoke on bone $[2,3]$. Smoking is associated with delayed fracture healing, reduced bone density, alterations in bone mineral content, and osteoporosis $[4,5]$. Recent data suggest that toxins contained in cigarette smoke may not only initiate and exacerbate tissue injury but also impair reparative processes via the initiation of inflammatory responses [6]. Tissue destruction may be exerted either through direct toxic effects (e.g., DNA damage), altered gene regulation, or indirectly through increased oxidative stress $[1,3,7]$. In our continuously ageing society this represents a major health problem, as quality of life is significantly reduced by prolonged hospital stays and reduced mobility.

Flavonoids are widely distributed in sources of vegetal origin (fruits, seeds, roots, flowers, tea, or wine). They are known to have multiple beneficial biological, pharmacological, and medicinal properties including anti-inflammatory, antimutagenic, antineoplastic, and cytoprotective effects [8]. A fundamental property of these molecules, responsible for many of their positive effects, is the antioxidant capacity. This is due to the presence of a series of structural characteristics that allow them to quelate ions of transition metals such as $\mathrm{Fe}^{2+}, \mathrm{Cu}^{2+}$ or $\mathrm{Zn}^{2+}$ and to catalyze the electron transport. Another important function is to scavenge reactive oxygen species (ROS) like superoxide anion, oxygen singlet, and lipidic peroxyradicals or to stabilize free ROS by means of hydrogenation or formation of complexes with oxidizing species [8]. Accordingly recent reports indicate the capacity to reduce and prevent bone loss and show positive effects on osteoblast and osteoclast activities [9, 10]. Among the more than 4000 varieties of the flavonoid family, Quercetin is an important member. It has been shown to reduce oxidative stress-dependent damage not only in vitro (e.g., ethanol-treated primary human hepatocytes) [11, 12] but also in vivo in rats exposed to methyl-mercury [13]. Moreover, recent evidence suggests that various flavonoids may positively influence negative effects of cigarette-associated tissue damage in humans [14].

The purpose of the here presented study was to determine whether Quercetin proved to exert a protective effect in osteoblasts exposed to CSM. In addition, this study's aim was to analyze the possible activation of antioxidative pathways by the flavonoid Quercetin in osteoblasts.

\section{MATERIALS AND METHODS}

Dulbecco's phosphate-buffered saline (DPBS) and cell culture medium and supplements were obtained from PAA Laboratories (Cölbe, Germany); antibodies from Santa Cruz Biotechnology (Heidelberg, Germany); and chemicals if not stated differently from Sigma (Munich, Germany).

\subsection{Isolation and Culture of Primary Human Osteoblasts}

Primary human osteoblasts were isolated from femur heads of patients undergoing total hip replacement. The isolation was approved by the ethical committee from the "Klinikum rechts der Isar", Technische Universität München. Briefly, cancellous bone was removed mechanically from the femur head and washed 
3-5 times with DPBS followed by $1 \mathrm{~h}$ incubation at $37^{\circ} \mathrm{C}$ with an equal volume of digestion buffer (DPBS, $0.07 \%$ collagenase II-Biochrom AG, Berlin, Germany). After digestion, cancellous bone was washed with culture medium (MEM/Ham's F12, 10\% FCS, $2 \mathrm{mM}$ L-glutamine, $100 \mathrm{U} / \mathrm{mL}$ penicillin, $100 \mu \mathrm{g} / \mathrm{mL}$ streptomycin, and $50 \mu \mathrm{M}$ L-ascorbate-2-phosphate, $50 \mu \mathrm{M} \beta$-glycerol-phosphate). Wash fraction was transferred to cell culture flasks to allow adherence of cells. Medium was changed every 4-5 days [15]. Osteoblasts were cultured and expanded until passage 3, where a pure population of osteoblasts, negative for CD14 and CD45 and positive for CD90 and CD105, was reached (flow cytometry). Then cells were plated for the experiments $\left(20,000\right.$ cells $\left./ \mathrm{cm}^{2}\right)$.

\subsection{Generation of CSM}

CSM was prepared from commercially available cigarettes (Marlboro, Philip Morris, Munich, Germany), as suggested by the Federal Trade Commission (FTC) and the International Organisation for Standardisation (ISO 10362-2). Briefly, the filter was removed and cigarettes were placed in a standard gas washing bottle (Lenz Laborglas GmbH \& Co.KG, Wertheim, Germany), subjected to negative pressure by using a peristaltic pump [16]. Here 3 cigarettes were bubbled through $25 \mathrm{~mL}$ of DMEM for $15 \mathrm{~min}$ at a rate of $1 \mathrm{puff} / \mathrm{min}$, each puff lasting for 2 seconds. The freshly prepared CSM was filtered $(0.22 \mu \mathrm{m}$ filter, Sarstedt, Nürnberg, Germany) before use. The concentration of CSM was determined and normalized by its optical density at $320 \mathrm{~nm}\left(\mathrm{OD}_{320}\right)$ in a plate reader (BMG Labtech, Offenburg, Germany).

\subsection{Determination of ROS}

After washing cells twice with DPBS they were incubated with $10 \mu \mathrm{M} 2^{\prime}, 7^{\prime}$-dichlorfluorescein-diacetate in serum-free culture medium for $30 \mathrm{~min}$ at $37^{\circ} \mathrm{C}$. After washing with DPBS the cells were stimulated with the smoke extract. After $15 \mathrm{~min}$ the fluorescence intensity $(\mathrm{ex} / \mathrm{em}=485 / 520 \mathrm{~nm})$ was quantified using a plate reader.

\subsection{Viability Measurement}

Viability was determined by resazurin conversion. Briefly, after stimulation $1 / 10$ volume of a $0.025 \%(\mathrm{w} / \mathrm{v})$ resazurin solution (in DPBS) was added to the cells. After $1 \mathrm{~h}$ incubation at $37^{\circ} \mathrm{C}$ fluorescence intensity was measured $(\mathrm{ex} / \mathrm{em}=540 / 590 \mathrm{~nm}$ ) using a plate reader and corrected to background control (solvent mixture without cells). Viability is given as $\%$ of control (untreated cells).

\subsection{Western Blot}

Cells were lysed in ice-cold RIPA lysis buffer $(50 \mathrm{mM}$ Tris; $250 \mathrm{mM} \mathrm{NaCl} ; 2 \%$ Nonidet-P40; $2.5 \mathrm{mM}$ EDTA; $0.1 \%$ SDS; $0.5 \%$ DOC; complete mini protease inhibitor and phosphatase inhibitor used according to the manufacturer's specifications; $\mathrm{pH}=7.2$ ). Protein concentration was determined by micro-Lowry. $30 \mu \mathrm{g}$ total protein was separated by SDS PAGE and transferred to nitrocellulose membranes (Carl Roth, Karlsruhe, Germany).

\subsection{Statistics}

Results are expressed as mean \pm SEM of at least 3 independent experiments $(N \geq 3)$ measured as triplicates or more $(n \geq 3)$. Data sets were compared by one-way analysis of variance followed by Bonferroni's multiple comparison test (GraphPad Prism Software, El Camino Real, USA). $P<0.05$ was taken as minimum level of significance. 


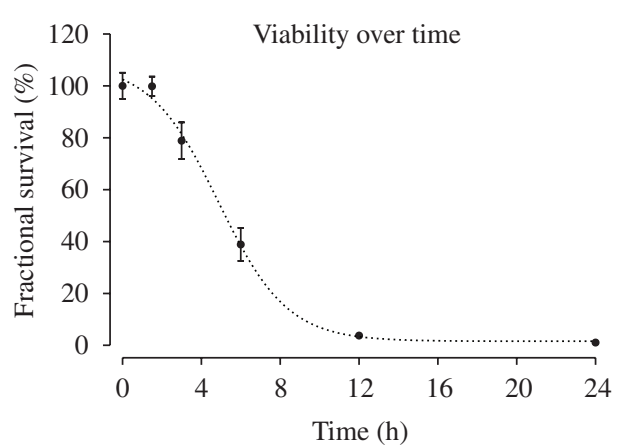

(a)

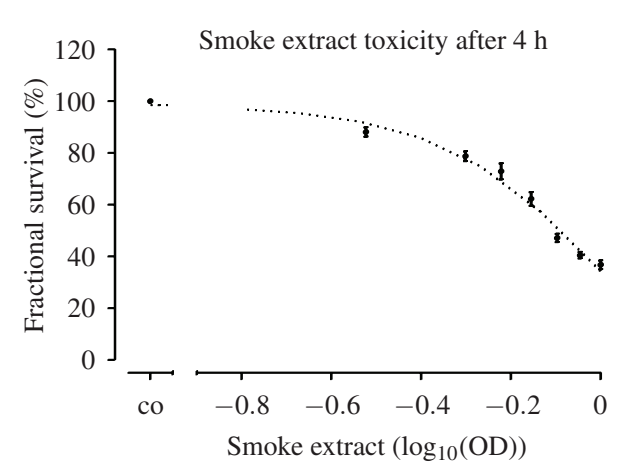

(c)

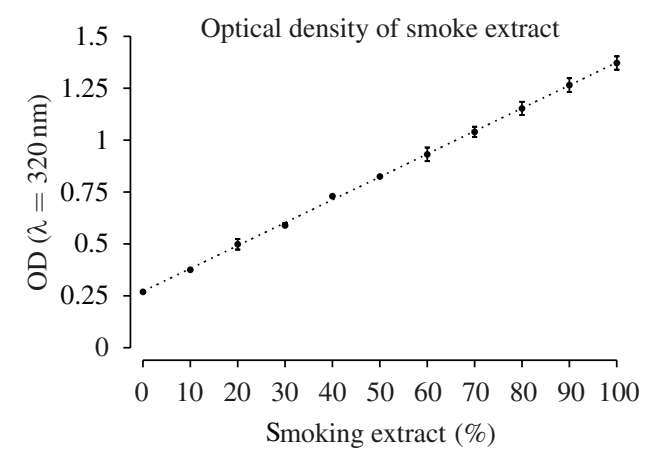

(b)

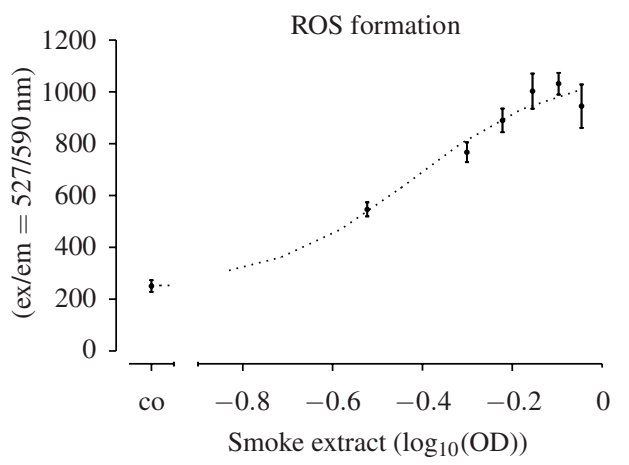

(d)

FIGURE 1: CSM damages primary human osteoblasts in a time- and concentration-dependent manner. (a) Primary human osteoblasts $(N=3, n=6)$ were treated with concentrated CSM for $0,1.5,3,6,12$, and $24 \mathrm{~h}$. After stimulation viability was determined by resazurin conversion. (b) The optical density of different dilutions $(0,10,20,30,40,50,60,70,80,90$, and $100 \%)$ of CSM was determined at $\lambda=320 \mathrm{~nm}\left(\mathrm{OD}_{320}\right)$. (c) The same dilutions were tested for their toxic effect after $4 \mathrm{~h}$ stimulation $\left(E C_{50} \approx 0.75 \mathrm{OD}_{320}\right)$. (d) After 15 min CSM induces significantly ( $P<0.01$ from $50 \%$ CSM on) the production of ROS in primary human osteoblasts $(N=3, n=4)$.

\section{RESULTS}

\subsection{CSM Reduces Viability of Osteoblasts Time- and Concentration-Dependently}

Primary human osteoblasts viability was determined after incubation for $0,1.5,3,6,12$, and $24 \mathrm{~h}$ with concentrated CSM $(N=3, n=6)$. Viability was reduced in a time-dependant manner, reaching $100 \%$ toxicity after $12 \mathrm{~h}$ and $50 \%$ toxicity ranging around $4 \mathrm{~h}$ (Figure 1(a)). In continuation and to standardize the experimental setup we identified the concentration of CSM when 50\% toxicity was reached after a $4 \mathrm{~h}$ incubation period $(N=3, n=6)$. This was obtained by a CSM with an $\mathrm{OD}_{320}$ of 0.8 (Figures 1 (b) and $1(\mathrm{c}))$.

\subsection{CSM Rapidly Induces ROS Formation in Osteoblasts Dose-Dependently}

Primary human osteoblasts $(N=3, n=4)$ loaded with DCFH-DA were exposed to different concentrations of CSM. After 15 min ROS formation was measured by an increase in fluorescence intensity. A significant increase in ROS formation was observed starting at an OD of $0.5(P<0.01 /$ Figure $1(\mathrm{~d}))$. 


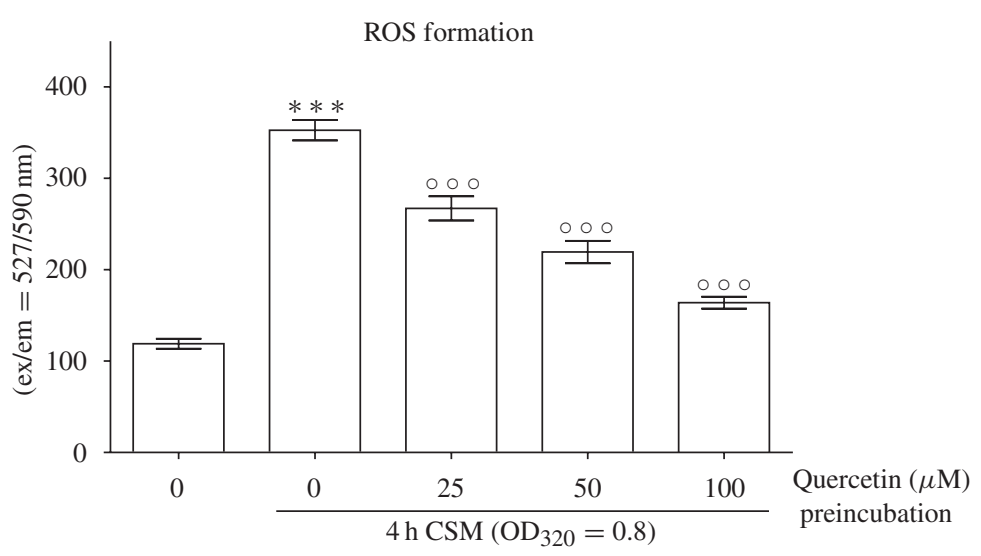

(a)

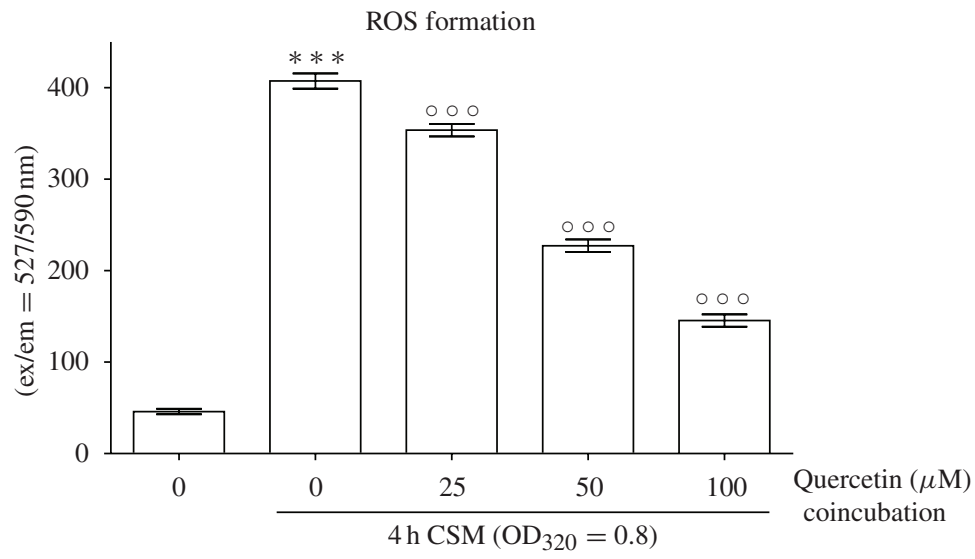

(b)

FIGURE 2: Quercetin reduces CSM-induced ROS production in primary human osteoblasts. (a) Preincubation of primary human osteoblasts $(N=3, n=4)$ with subtoxic concentrations of Quercetin $(25,50$, $100 \mu \mathrm{M})$ reduces the formation of ROS after 15 min treatment with $C S M\left(O_{320}=0.8\right)$. (b) Similar results were observed during coincubation. ${ }^{* * *} P<0.001$ compared to untreated cells, ${ }^{\circ 00} P<0.001$ compared to CSM-treated cells.

\subsection{Quercetin Reduces ROS Production from CSM in Primary Human Osteoblasts}

We determined the $\mathrm{EC}_{50 / 24 \mathrm{~h}}$ of Quercetin to be $280.1 \pm 1.1 \mu \mathrm{M}(N=3, n=4)$. Coincubation with subtoxic doses of Quercetin $(25,50,100 \mu \mathrm{M})$ significantly reduced ROS formation dose-dependently $(N=3$, $n=4 /$ Figure 2(a)). Concordantly, similar results were seen for pre-incubation with Quercetin $(N=3$, $n=4 /$ Figure 2(b)).

\subsection{Quercetin Protects Primary Human Osteoblasts from CSM-Dependent Damage}

We measured viability of CSM-treated $\left(\mathrm{OD}_{320}=0.8\right)$ primary human osteoblasts after $4 \mathrm{~h}$ pre-, co-, and post-incubation with subtoxic concentrations of Quercetin $(25,50$, and $100 \mu \mathrm{M})$. An improved viability was achieved through all three setups showing the most beneficial results for coincubation reaching nearly $100 \%$ survival at a concentration of $100 \mu \mathrm{M}$ Quercetin. Interestingly, post-incubation with Quercetin also induced recovery of primary human osteoblasts up to $80 \%$ of untreated cells. Noteworthy, preincubation established a similar protective effect with a cell survival of nearly $75 \%$ (Figure 3 ). 


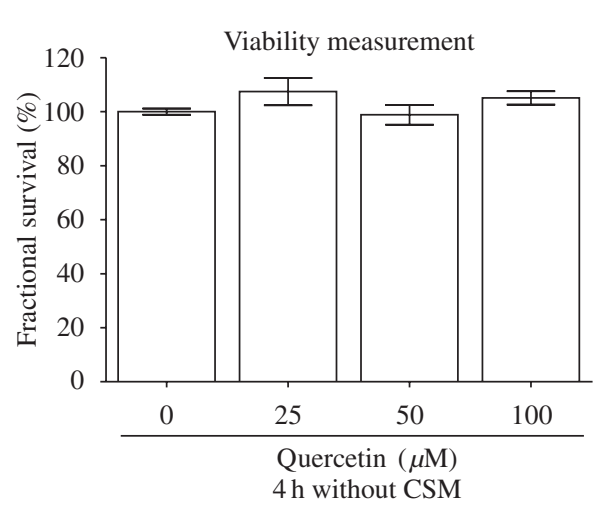

(a)

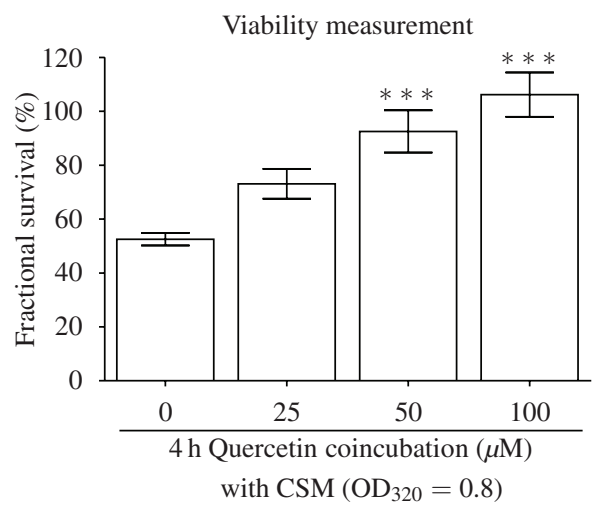

(c)

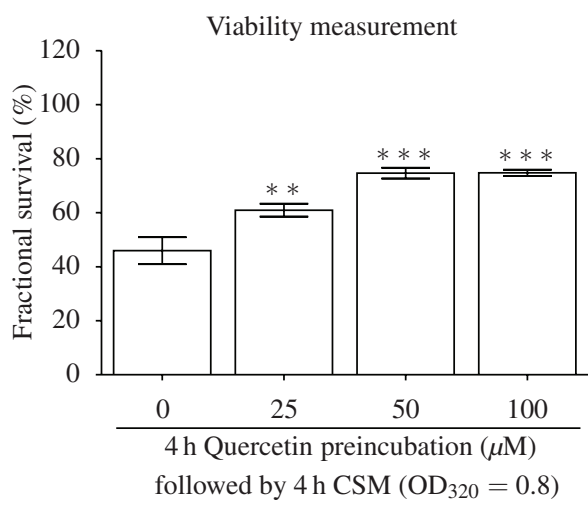

(b)

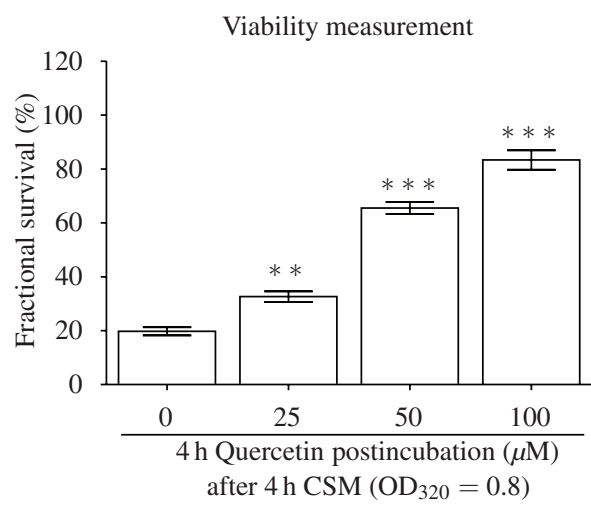

(d)

FIGURE 3: Quercetin reduces CSM-induced cellular damage in primary human osteoblasts dosedependently. (a) Stimulation of primary human osteoblasts $(N=3, n=4)$ for $4 \mathrm{~h}$ with 25,50 , and $100 \mu \mathrm{M}$ Quercetin is not toxic to the cells. (b) Pre-, (c) co-, and (d) postincubation with these subtoxic concentrations of Quercetin protects primary human osteoblasts $(N=3, n=4)$ from CSM-induced $\left(\mathrm{OD}_{320}=0.8\right)$ cellular damage. Viability was measured by resazurin conversion. Results are given as fractional survival [\%] compared to untreated cells. ${ }^{* *} P<0.01,{ }^{* * *} P<0.001$ compared to CSM-treated cells.

\subsection{Quercetin Induces Expression of HO-1 and SOD-1 in Human Osteoblasts}

Primary human osteoblasts exposed to $100 \mu \mathrm{M}$ Quercetin for $4 \mathrm{~h}$ were examined for expression of the antioxidative enzymes HO-1 and SOD-1, the transcription factor pNrf2 and phosphporylation of ERK and SAPK/JNK by Western blot. An increase in pNrf2, HO-1, and SOD-1 protein levels became evident when stimulated with Quercetin. At the same time phosphorylation of ERK1/2 increased but not phosphorylation of SAPK/JNK (Figure 4(a)).

\subsection{Inhibition of HO-1 Reverses Protective Effects of Quercetin}

We determined the $\mathrm{EC}_{50 / 24 \mathrm{~h}}$ of the HO-1 inhibitor zinc protoporphyrin (ZnPP9) to be $12.3 \pm 1.1 \mu \mathrm{M}$ $(N=3, n=4)$. In order to investigate if the protective effect of Quercetin is dependent on HO-1 expression we measured the survival of CSM-treated primary human osteoblasts after pre-, co-, and post-incubation with $100 \mu \mathrm{M}$ Quercetin in the presence or absence of $10 \mu \mathrm{M} \mathrm{ZnPP9}(N=3, n=4)$. It became evident that the presence of ZnPP9 diminished the protective effect of Quercetin (Figure 4(b)). 


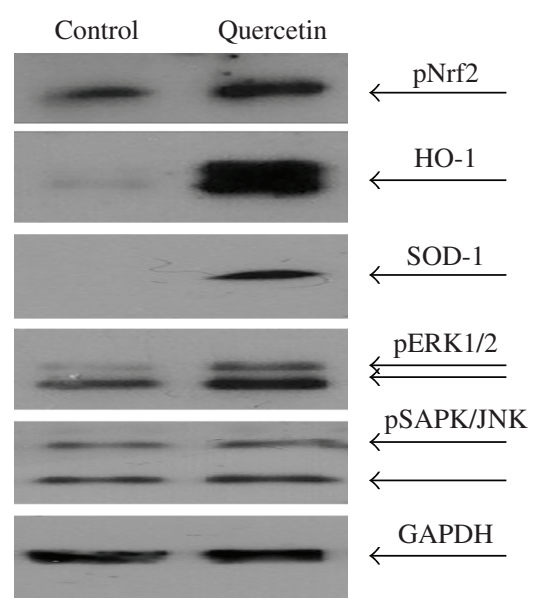

(a)

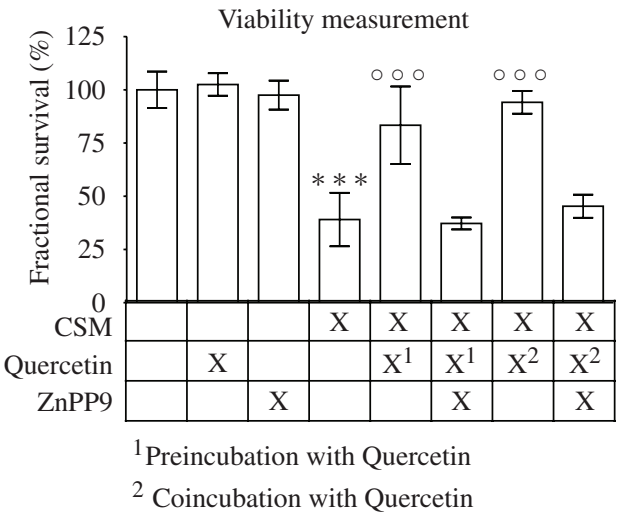

(b)

FIGURE 4: Quercetin protects primary human osteoblasts from CSM-induced damage via upregulation of anti-oxidative enzymes HO-1 and SOD-1. (a) Representative picture of a Western blot for pNrf2, HO-1, SOD-1, pERK1/2, and pSAPK in primary human osteoblasts treated with or without $100 \mu \mathrm{M}$ Quercetin. Quercetin induces the expression of HO-1 and SOD-1 and phosphorylation of pNrf2 and ERK1/2. GAPDH was used as loading control. (b) The HO-1 inhibitor ZnPP9 $(10 \mu \mathrm{M})$ reduces the protective effect of Quercetin $(100 \mu \mathrm{M} /$ pre- and co-incubation) on primary human osteoblasts $(N=3, n=4)$ treated with CSM $\left(\mathrm{OD}_{320}=0.8\right)$ for $4 \mathrm{~h} .{ }^{* * *} P<0.001$ compared to untreated cells; ${ }^{\circ}{ }^{\circ} P<0.001$ compared to CSM-treated cells.

\section{DISCUSSION AND CONCLUSIONS}

Our results clearly demonstrate the toxic effects of CSM on primary human osteoblasts. An increase in oxidative stress is related to osteoblasts cell death. According to recent experimental findings this plays a key role in clinical observations such as delayed fracture healing, increased rate of osteoporosis, or reduced bone mass [2-5]. Quercetin is one of the most frequently studied flavonoids, and it exerts beneficial but also deleterious effects depending on the cell type [8-10]. There have been reports indicating the inhibitory potential of Quercetin on osteoclasts via its estrogenic effect acting as a phytoestrogen in rabbit long bones. Induction of osteoclasts apoptosis and antiresorptive actions is, concerning this matter, attributable to Quercetin [10]. Pang and coworkers complement these findings [9]. Despite these results the precise molecular mechanisms of Quercetin remain uncertain. Nam and colleagues for example showed that Quercetin at a concentration as low as $20 \mu \mathrm{M}$ induced apoptotic cell death in the mouse osteoblastic cell line MC3T3-E1 in an ERK1/2-dependent manner [17]. These data are at variance with previous reports in which Quercetin does not induce cell death in rat calvaria osteoblastic cells [18], MC3T3-E1 cells [19], and MG-63 human osteoblastic cells [20]. In our model Quercetin induced phosphorylation of ERK1/2; however, reduced cell viability was only observed after $24 \mathrm{~h}$ exposure at concentrations above $200 \mu \mathrm{M}$. Highlighting the discrepancy to the findings of Nam and colleagues we obtained significant protective effects of Quercetin at concentrations comparable to physiological relevant plasma levels. Furthermore, the ability to reduce ROS was not observed at concentrations below $50 \mu \mathrm{M}$ in osteoclasts as described by Wattel and coworkers [10]. However, even concentrations as low as $25 \mu \mathrm{M}$ of Quercetin protect primary human hepatocytes from ethanol-induced damage $[11,12]$.

Nevertheless, despite our observed positive effects, it is mandatory to remain cautious when analyzing its clinical potential. There have been reports of DNA damage at Quercetin concentrations of $>30 \mu \mathrm{M}$ [21]. Furthermore, Quercetin concentrations exceeding $75 \mu \mathrm{M}$ can have some intrinsic cytotoxicity in cultured renal tubular cells [22]. High doses may even exert genotoxic effects [23]. Moreover, it has been reported that Quercetin at concentrations $>50 \mu \mathrm{M}$ is able to participate in the oxidation of 
NADPH in liver cells, shifting the cellular conditions to a more oxidized state [24]. An additional aspect is the limited bioavailability of flavonoids, due to its low absorption and rapid elimination [8]. Thus, absorbed Quercetin becomes rapidly conjugated in the human body. The group of Chattopadhyay could show that a Quercetin C-glucoside improved bone formation rate and trabecular microarchitecture in ovariectomized rats better than Quercetin itself $[25,26]$.

Using the here presented in vitro model we emphasized the potent protective effect of Quercetin in osteoblasts in pre-, co-, and post-incubation conditions. Our data is in concordance with recent epidemiological and experimental findings demonstrating positive effects of flavonoids on bone. Mühlbauer and colleagues have shown that several vegetables significantly inhibited bone resorption in rats [27]. Highlighting flavonoids beneficial effects Horcajada-Molteni reduced bone resorption in vivo in ovariectomized rats through rutin, a Quercetin glycoside [28]. Furthermore, Arjmandi and coworkers showed that dietary soybean protein prevents bone loss in an ovariectomized rat model of osteoporosis [29]. Beside this experimental evidence, a recent paper by Bohn and coworkers clearly suggests that cellular stress defense is positively modulated by antioxidant-rich food in human cigarette smokers [14].

We have shown that Quercetin can induce the antioxidant enzyme HO-1 via its transcription factor pNRF2, thus protecting primary human osteoblasts in the preincubation setting. A similar mechanism has been observed in primary human hepatocytes $[11,12]$. This antioxidant activity primes the cells against radicals possibly causing cell death. This appears to be of great interest in preoperative treatment of patients in order to diminish stress-related toxicity. Flavonoids directly reduce oxidative stress in cells by their structural properties as radical scavengers, most probably related to the phenolic hydroxyl groups attached to the ring structures. This, in combination with upregulation of HO-1, might explain the more protective effects of Quercetin during the co- and postincubation setup. This elementary function could be used to treat patients already exposed to elevated ROS levels, since ROS have been suggested to be involved in the pathogenesis of bone loss-related diseases [30].

In summary, our results show that Quercetin, a natural flavonoid widely distributed in a healthy human diet, is able to reduce ROS formation. It aids the antioxidant network in compensating the elevated redox imbalance in patients with increased ROS levels such as smokers. Furthermore, Wattel et al. showed inhibitory effects of Quercetin on bone resorption by inhibiting osteoclast activity [10].

In combination with these findings our data suggest that Quercetin may not only exert a beneficial effect in bone remodeling via its influence on osteoclasts, but also via a protective effect on osteoblasts. Therefore, Quercetin might be used in not only supporting bone regeneration in smokers but also strengthening the bone metabolism in different pathogenesis. Quercetin can support the maintenance of skeletal integrity while buffering the toxic effect of CSM. Further research in this field (e.g., human trials) is mandatory to elucidate possible therapeutic strategies.

\section{ABBREVIATIONS}

CSM: Cigarette smoke medium

ROS: Reactive oxygen species

HO-1: Heme-oxygenase-1

SOD-1: Superoxide-dismutase-1.

\section{ACKNOWLEDGMENTS}

The authors like to thank Fritz Seidl for providing language help and Marina Unger and Ursula Hopfner for their excellent technical assistance. Karl F. Braun and Sabrina Ehnert contributed equally to this work. 


\section{REFERENCES}

[1] D. E. Rothem, L. Rothem, M. Soudry, A. Dahan, and R. Eliakim, "Nicotine modulates bone metabolismassociated gene expression in osteoblast cells," Journal of Bone and Mineral Metabolism, vol. 27, no. 5, pp. 555-561, 2009.

[2] M. P. Akhter, A. D. Lund, and C. G. Gairola, "Bone biomechanical property deterioration due to tobacco smoke exposure," Calcified Tissue International, vol. 77, no. 5, pp. 319-326, 2005.

[3] J. B. Cesar-Neto, P. M. Duarte, M. C. G. de Oliveira, C. H. Tambeli, E. A. Sallum, and F. H. Nociti Jr., "Smoking modulates interleukin-6:interleukin-10 and RANKL: osteoprotegerin ratios in the periodontal tissues," Journal of Periodontal Research, vol. 42, no. 2, pp. 184-191, 2007.

[4] P. B. Rapuri, J. C. Gallagher, K. E. Balhorn, and K. L. Ryschon, "Smoking and bone metabolism in elderly women," Bone, vol. 27, no. 3, pp. 429-436, 2000.

[5] S. W. N. Ueng, S. S. Lin, C. R. Wang, S. J. Lin, C. L. Tai, and C. H. Shih, "Bone healing of tibial lengthening is delayed by cigarette smoking: study of bone mineral density and torsional strength on rabbits," Journal of Trauma, vol. 46, no. 1, pp. 110-115, 1999.

[6] H. Wang, X. Liu, T. Umino et al., "Cigarette smoke inhibits human bronchial epithelial cell repair processes," American Journal of Respiratory Cell and Molecular Biology, vol. 25, no. 6, pp. 772-779, 2001.

[7] A. P. Giorgetti, J. B. Cesar-Neto, K. G. Ruiz, M. Z. Casati, E. A. Sallum, and F. H. Nociti Jr., "Cigarette smoke inhalation modulates gene expression in sites of bone healing: a study in rats," Oral Surgery, Oral Medicine, Oral Pathology, Oral Radiology and Endodontics, vol. 110, pp. 447-452, 2011.

[8] J. Gonzalez-Gallego, M. V. Garcia-Mediavilla, S. Sanchez-Campos, and M. J. Tunon, "Fruit polyphenols, immunity and inflammation," British Journal of Nutrition, vol. 104, supplement 3, pp. S15-S27, 2011.

[9] J. L. Pang, D. A. Ricupero, S. Huang et al., "Differential activity of kaempferol and quercetin in attenuating tumor necrosis factor receptor family signaling in bone cells," Biochemical Pharmacology, vol. 71, no. 6, pp. 818-826, 2006.

[10] A. Wattel, S. Kamel, C. Prouillet et al., "Flavonoid quercetin decreases osteoclastic differentiation induced by RANKL via a mechanism involving NF kappa B and AP-1," Journal of Cellular Biochemistry, vol. 92, no. 2, pp. 285-295, 2004.

[11] S. Liu, W. Hou, P. Yao et al., "Quercetin protects against ethanol-induced oxidative damage in rat primary hepatocytes," Toxicol in Vitro, vol. 24, pp. 516-522, 2011.

[12] P. Yao, A. Nussler, L. Liu et al., "Quercetin protects human hepatocytes from ethanol-derived oxidative stress by inducing heme oxygenase-1 via the MAPK/Nrf2 pathways," Journal of Hepatology, vol. 47, no. 2, pp. 253-261, 2007.

[13] G. R. Barcelos, D. Grotto, J. M. Serpeloni et al., "Protective properties of quercetin against DNA damage and oxidative stress induced by methylmercury in rats," Archives of Toxicology, vol. 85, no. 9, pp. 1151-1157, 2011.

[14] S. K. Bohn, M. C. Myhrstad, M. Thoresen et al., "Blood cell gene expression associated with cellular stress defense is modulated by antioxidant-rich food in a randomised controlled clinical trial of male smokers," $B M C$ Medicine, vol. 8, article 54, 2010.

[15] S. Ehnert, J. Baur, A. Schmitt et al., “TGF-beta1 as possible link between loss of bone mineral density and chronic inflammation," PLoS One, vol. 5, no. 11, Article ID e14073, 2010.

[16] S. Lin, V. Tran, and P. Talbot, "Comparison of toxicity of smoke from traditional and harm-reduction cigarettes using mouse embryonic stem cells as a novel model for preimplantation development," Human Reproduction, vol. 24, no. 2, pp. 386-397, 2009.

[17] T. W. Nam, C. I. Yoo, H. T. Kim, C. H. Kwon, J. Y. Park, and Y. K. Kim, “The flavonoid quercetin induces apoptosis and inhibits migration through a MAPK-dependent mechanism in osteoblasts," Journal of Bone and Mineral Metabolism, vol. 26, no. 6, pp. 551-560, 2008.

[18] M. Notoya, Y. Tsukamoto, H. Nishimura et al., "Quercetin, a flavonoid, inhibits the proliferation, differentiation, and mineralization of osteoblasts in vitro," European Journal of Pharmacology, vol. 485, no. 1-3, pp. 89-96, 2004.

[19] Y. O. Son, S. H. Kook, K. C. Choi et al., "Quercetin accelerates TNF-alpha-induced apoptosis of MC3T3-E1 osteoblastic cells through caspase-dependent and JNK-mediated pathways," European Journal of Pharmacology, vol. 579, no. 1-3, pp. 26-33, 2008. 
[20] C. Prouillet, J. C. Maziere, C. Maziere, A. Wattel, M. Brazier, and S. Kamel, "Stimulatory effect of naturally occurring flavonols quercetin and kaempferol on alkaline phosphatase activity in MG-63 human osteoblasts through ERK and estrogen receptor pathway," Biochemical Pharmacology, vol. 67, no. 7, pp. 1307-1313, 2004.

[21] M. J. Laughton, B. Halliwell, P. J. Evans, and J. R. S. Hoult, "Antioxidant and pro-oxidant actions of the plant phenolics quercetin, gossypol and myricetin. Effects on lipid peroxidation, hydroxyl radical generation and bleomycin-dependent damage to DNA," Biochemical Pharmacology, vol. 38, no. 17, pp. 2859-2865, 1989.

[22] M. K. Kuhlmann, E. Horsch, G. Burkhardt, M. Wagner, and H. Kohler, "Reduction of cisplatin toxicity in cultured renal tubular cells by the bioflavonoid quercetin," Archives of Toxicology, vol. 72, no. 8, pp. 536-540, 1998.

[23] J. da Silva, S. M. Herrmann, V. Heuser et al., "Evaluation of the genotoxic effect of rutin and quercetin by comet assay and micronucleus test," Food and Chemical Toxicology, vol. 40, no. 7, pp. 941-947, 2002.

[24] G. D. Buss, J. Constantin, L. C. N. de Lima et al., "The action of quercetin on the mitochondrial NADH to $\mathrm{NAD}(+)$ ratio in the isolated perfused rat liver," Planta Medica, vol. 71, no. 12, pp. 1118-1122, 2005.

[25] K. Sharan, J. S. Mishra, G. Swarnkar et al., "A novel quercetin analogue from a medicinal plant promotes peak bone mass achievement and bone healing after injury and exerts an anabolic effect on osteoporotic bone: the role of aryl hydrocarbon receptor as a mediator of osteogenic action," Journal of Bone and Mineral Research, vol. 26, pp. 2096-2111, 2011.

[26] J. A. Siddiqui, G. Swarnkar, K. Sharan et al., "A naturally occurring rare analog of quercetin promotes peak bone mass achievement and exerts anabolic effect on osteoporotic bone," Osteoporosis International, no. 12, pp. 3013-3027, 2011.

[27] R. C. Muhlbauer, A. Lozano, A. Reinli, and H. Wetli, "Various selected vegetables, fruits, mushrooms and red wine residue inhibit bone resorption in rats," Journal of Nutrition, vol. 133, no. 11, pp. 3592-3597, 2003.

[28] M. N. Horcajada-Molteni, V. Crespy, V. Coxam, M. J. Davicco, C. Remesy, and J. P. Barlet, "Rutin inhibits ovariectomy-induced osteopenia in rats," Journal of Bone and Mineral Research, vol. 15, no. 11, pp. 2251-2258, 2000.

[29] B. H. Arjmandi, L. Alekel, B. W. Hollis et al., "Dietary soybean protein prevents bone loss in an ovariectomized rat model of osteoporosis," Journal of Nutrition, vol. 126, no. 1, pp. 161-167, 1996.

[30] S. Basu, K. Michaelsson, H. Olofsson, S. Johansson, and H. Melhus, "Association between oxidative stress and bone mineral density," Biochemical and Biophysical Research Communications, vol. 288, pp. 275-279, 2001.

\section{This article should be cited as follows:}

Karl F. Braun, Sabrina Ehnert, Thomas Freude, José T. Egaña, Thilo L. Schenck, Arne Buchholz, Andreas Schmitt, Sebastian Siebenlist, Lilianna Schyschka, Markus Neumaier, Ulrich Stöckle, and Andreas K. Nussler, "Quercetin Protects Primary Human Osteoblasts Exposed to Cigarette Smoke through Activation of the Antioxidative Enzymes HO-1 and SOD-1," TheScientificWorldJOURNAL, vol. 11, pp. 2348-2357, 2011. 

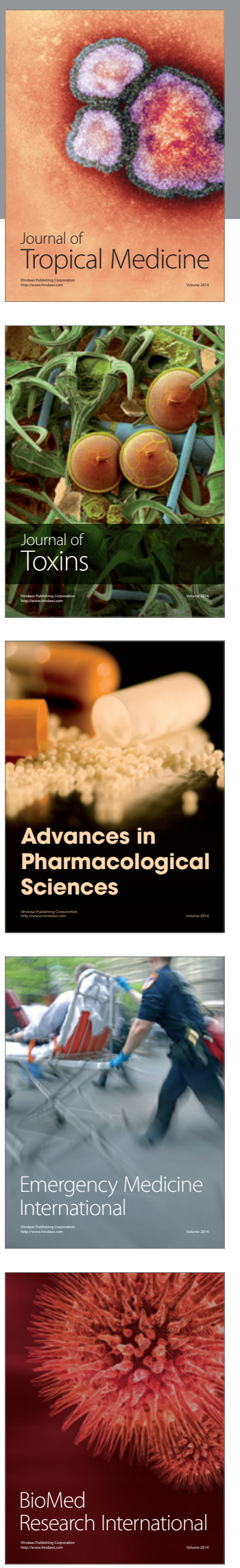
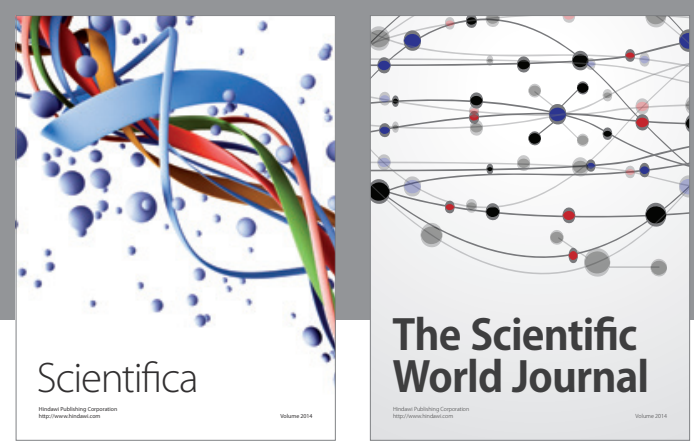

The Scientific World Journal
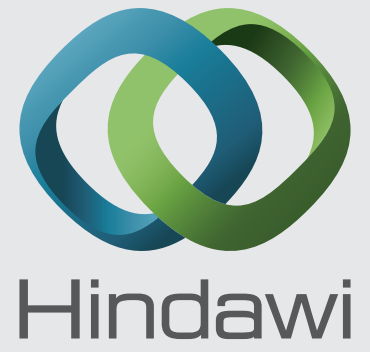

Submit your manuscripts at

http://www.hindawi.com
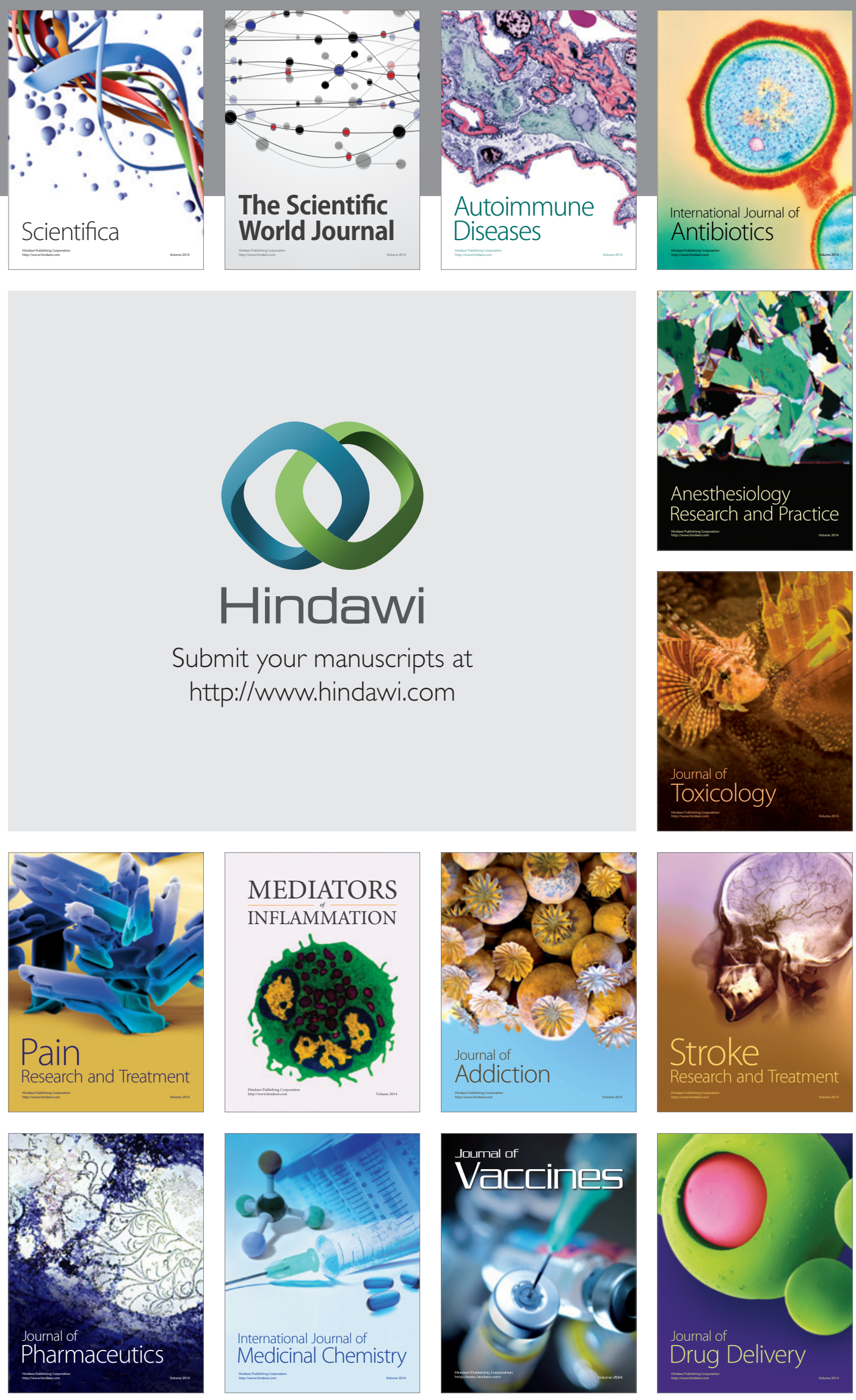